\title{
ON A PROBLEM OF S. MAZUR
}

\author{
LÁSZLÓ SZÉKELYHIDI
}

\begin{abstract}
In this work a generalization of Mazur's problem concerning the continuity of linear functionals is given.
\end{abstract}

S. Mazur asked (about 1935) the following question [7, Problem 24]: In a Banach space $E$ an additive functional $f$ is given with the property that, for any continuous function $\varphi:[0,1] \rightarrow E$ the function $f \circ \varphi$ is Lebesguemeasurable. Is $f$ continuous? This question was answered affirmatively by I. Labuda and R. D. Mauldin in [3] by the following theorem:

Theorem 1. Let $E$ be a Banach space, $F$ a Hausdorff topological vector space, $f: E \rightarrow F$ an additive operator. If $f \circ \varphi$ is Lebesgue-measurable for any continuous function $\varphi:[0,1] \rightarrow E$, then $f$ is continuous.

The following more general theorem is due to Z. Lipecki [4].

Theorem 2. Let $G, H$ be Hausdorff topological abelian groups, $G$ is metrizable, complete, connected and locally arcwise connected, and let $f: G \rightarrow H$ be a homomorphism. If $f \circ \varphi$ is Lebesgue-measurable for any continuous function $\varphi:[0,1] \rightarrow G$, then $f$ is continuous.

Recently, R. Ger presented similar results concerning convex functionals [2]. The aim of this paper is to give another generalization of Mazur's problem. Namely, we show, that if in the original problem $f$ is an exponential polynomial, then the statement remains valid.

First we collect some necessary facts about polynomials and exponential polynomials on groups. Most of these results can be found in [5,6]. Let $G$ be an abelian group, $H$ a complex linear space. The function $p: G \rightarrow H$ is called a polynomial if for some nonnegative integer $N$ we have

$$
\Delta_{y_{1}, \ldots, y_{N+1}}^{N+1} p(x)=0
$$

for all $x, y_{1}, \ldots, y_{N+1}$ in $G$. The smallest integer $N$ with this property is called the degree of $p$ and is denoted by $\operatorname{deg} p$. It is well known [1] that any

Received by the editors April 1, 1988.

1980 Mathematics Subject Classification (1985 Revision). Primary 22B05; Secondary 46B99, 46E99.

This work is supported by a research fellowship given by the Alexander von Humboldt Foundation. 
function $p$ satisfying (1) can uniquely be represented in the form

$$
p(x)=A_{N}(x, \ldots, x)+A_{N-1}(x, \ldots, x)+\cdots+A_{1}(x)+A_{0}
$$

for all $x$ in $G$, where $A_{k}: G^{k} \rightarrow H$ is a $k$-additive and symmetric function $(k=1,2, \ldots, N)$ and $A_{0}$ is in $H$. For the sake of simplicity we shall use the notation

$$
A^{(k)}(x)=A_{k}(x, \ldots, x)
$$

for all $x$ in $G$, that is $A^{(k)}$ is the diagonalization of the $k$-additive and symmetric function $A_{k},(k=1,2, \ldots, N)$.

Let $C$ denote the set of complex numbers. The function $m: G \rightarrow C$ is called an exponential if for all $x, y$ in $G$ we have

$$
m(x+y)=m(x) m(y)
$$

and $m$ is not identically zero. That is, exponentials are just the homomorphisms of $G$ into the multiplicative group of nonzero complex numbers.

The function $f: G \rightarrow H$ is called an exponential polynomial if it has a representation

$$
f(x)=\sum_{k=1}^{n} p_{k}(x) m_{k}(x)
$$

for all $x$ in $G$, where $p_{k}: G \rightarrow H$ is a polynomial and $m_{k}: G \rightarrow C$ is an exponential $(k=1, \ldots, n)$. It is well known [6] that if in (2) we have $m_{i} \neq m_{j}$ for $i \neq j$ then the representation (2) for $f$ is unique.

In order to prove our main theorem for exponential polynomials we first consider the case of polynomials.

Theorem 3. Let $G$ be a metrizable topological abelian group which is complete, connected and locally arcwise connected, further let $H$ be a metrizable locally convex complex topological vector space and $p: G \rightarrow H$ a polynomial. If $p \circ \varphi$ is Lebesgue-measurable for any continuous function $\varphi:[0,1] \rightarrow G$, then $p$ is continuous.

Proof. Let $p=A^{(N)}+q$, where $N \geq 1$ is an integer, $A_{N}: G^{N} \rightarrow H$ is $N$ additive and symmetric and $q: G \rightarrow H$ is a polynomial of degree at most $N-1$. It is enough to show that $A_{N}$ is continuous, then by induction we have the statement. It is well known [1] that

$$
\begin{aligned}
& A_{N}\left(x_{1}, x_{2}, \ldots, x_{N}\right)=\frac{1}{N !} \Delta_{x_{1}, x_{2} \ldots \ldots, x_{N}}^{N} p(0) \\
& \quad=\frac{1}{N !} \sum_{i_{1}<\cdots<i_{k}}(-1)^{N-k} p\left(x_{i_{1}}+\cdots+x_{i_{k}}\right)
\end{aligned}
$$

which implies that the function $t \rightarrow A_{N}\left(\varphi(t), x_{2}, \ldots, x_{N}\right)$ is Lebesgue-measurable for any continuous function $\varphi:[0,1] \rightarrow G$, and for any fixed $x_{2}, \ldots, x_{N}$ in $G$. Using the symmetry of $A_{N}$ and Theorem 2 we have that $A_{N}$ is continuous in each variable. From the theorem of Baire it follows that $A_{N}$ is 
continuous at least at one point. Then, using the connectedness of $G$, it follows from Theorem 4.2 in [5] that $A_{N}$ is continuous.

Theorem 4. Let $G$ be a metrizable topological abelian group which is complete, connected and locally arcwise connected, further let $H$ be a metrizable locally convex complex topological vector space and $f: G \rightarrow H$ an exponential polynomial. If $f \circ \varphi$ is Lebesgue-measurable for any continuous function $\varphi:[0,1] \rightarrow G$, then $f$ is continuous.

Proof. Let $f=\sum_{k=1}^{n} p_{k} m_{k}$, where $n \geq 1$ is an integer, $p_{k}: G \rightarrow H$ is a polynomial and $m_{k}: G \rightarrow C$ is an exponential $(k=1,2, \ldots, n), m_{i} \neq m_{j}$ for $i \neq j$ and $p_{k}=A_{k}^{\left(N_{k}\right)}+q_{k}$, where $A_{k, N_{k}}: G^{N_{k}} \rightarrow H$ is $N_{k}$-additive and symmetric, $q_{k}: G \rightarrow H$ is a polynomial of degree at most $N_{k}-1, A_{k, N_{k}} \neq 0$ $(k=1,2, \ldots, n)$. We show that $m_{k}, A_{k}^{\left(N_{k}\right)}$ is continuous $(k=1,2, \ldots, n)$. By induction on $n$, first let $n=1, f=p_{1} m_{1}$. Here we use induction on the degree of $p_{1}$. If $\operatorname{deg} p_{1}=0$, then $p_{1}$ is constant and $p_{1} \neq 0$. It is very easy to see, that in this case the property of $f$ implies that $m_{1} \circ \varphi$ is Lebesguemeasurable for any continuous function $\varphi:[0,1] \rightarrow G$, hence by Theorem 2 , $m_{1}$ is continuous. Then $p_{1} \circ \varphi$ is Lebesgue-measure for any continuous function $\varphi:[0,1] \rightarrow G$, and by Theorem $3, p_{1}$ is continuous and hence $f$ is continuous. If $\operatorname{deg} p_{1} \geq 1$ then $p_{1}$ is nonconstant, hence there exists $y$ for which $\Delta_{y} p_{1}$ is not identically zero and $\operatorname{deg} \Delta_{y} p_{1}<\operatorname{deg} p_{1}$. On the other hand

$$
\begin{aligned}
\Delta_{y} p_{1}(x) m_{1}(x) & =m_{1}(-y) p_{1}(x+y) m_{1}(x+y)-p_{1}(x) m_{1}(x) \\
& =m_{1}(-y) f(x+y)-f(x)
\end{aligned}
$$

that is the function $\left(\Delta_{y} p_{1} m_{1}\right) \circ \varphi$ is Lebesgue-measurable for any continuous function $\varphi:[0,1] \rightarrow G$ which implies the statement of the theorem for $n=1$. Let now $n \geq 2$. We show, that, for example, $m_{2}$ and $A_{2}^{\left(N_{2}\right)}$ are continuous. Let $y$ be an element for which $m_{1}(y) \neq m_{2}(y)$. Then we have

$$
\Delta_{y}^{N_{1}+1}\left(f m_{1}^{-1}\right)(x)=m_{1}(x)^{-1} \sum_{j=0}^{N_{1}+1}\left(\begin{array}{c}
N_{1}+1 \\
j
\end{array}\right)(-1)^{N_{1}+1-j} m_{1}(y)^{-j} f(x+j y)
$$

by the definition of difference operators. From this equation we infer that the function $\left[m_{1} \Delta_{y}^{N_{1}+1}\left(f m_{1}^{-1}\right)\right] \circ \varphi$ is Lebesgue-measurable for any continuous function $\varphi:[0,1] \rightarrow G$, by the same property of $f$. On the other hand

$$
\left(f m_{1}^{-1}\right)(x)=p_{1}(x)+\sum_{k=2}^{n} p_{k}(x)\left(m_{k} m_{1}^{-1}\right)(x)
$$


holds for all $x$ in $G$. By taking differences we have

$$
\begin{aligned}
& \Delta_{y}^{N_{1}+1}\left(f m_{1}^{-1}\right)(x)=\sum_{k=2}^{n} \Delta_{y}^{N_{1}+1}\left[\left(m_{k} m_{1}^{-1}\right)\left(A_{k}^{\left(N_{k}\right)}+q_{k}\right)\right](x) \\
& =\sum_{k=2}^{n} \sum_{j=0}^{N_{1}+1}\left(\begin{array}{c}
N_{1}+1 \\
j
\end{array}\right)(-1)^{N_{1}+1-j} m_{k}(x) m_{1}(x)^{-1} m_{k}(y)^{j} m_{1}(y)^{-j} \\
& \times\left(A_{k}^{\left(N_{k}\right)}(x+j y)+q_{k}(x+j y)\right) \\
& =m_{1}(x)^{-1} \sum_{k=2}^{n} m_{k}(x)\left[\sum_{j=0}^{N_{1}+1}\left(\begin{array}{c}
N_{1}+1 \\
j
\end{array}\right)(-1)^{N_{1}+1-j} m_{k}(y)^{j}\right. \\
& \left.\times m_{1}(y)^{-j}\left(A_{k}^{\left(N_{k}\right)}(x)+q_{k, j, y}^{*}(x)\right)\right] \\
& =m_{1}(x)^{-1} \sum_{k=2}^{n} m_{k}(x)\left[\left(m_{k}(y) m_{1}(y)^{-1}-1\right)^{N_{1}+1} A_{k}^{\left(N_{k}\right)}(x)+q_{k, y}^{*}(x)\right],
\end{aligned}
$$

where $q_{k, j, y}^{*}: G \rightarrow H$ is a polynomial of degree at most $N_{k}-1$ and $q_{k, y}^{*}: G \rightarrow$ $H$ is a polynomial of degree at most $N_{k}-1 \quad\left(k=2, \ldots, n ; j=0,1, \ldots, N_{1}+\right.$ 1). We have a representation for the exponential polynomial $m_{1} \Delta_{y}^{N_{1}+1}\left(f m_{1}^{-1}\right)$ from which we infer-by the above consideration-that $m_{k}$ and its polynomial coefficient is continuous. It follows that $\left(m_{k}(y) m_{1}(y)^{-1}-1\right)^{N_{1}+1} A_{k}^{\left(N_{k}\right)}$ must be continuous, and especially -as $m_{2}(y) \neq m_{1}(y)$ 一the function $A_{2}^{\left(N_{2}\right)}$ is continuous. Hence the theorem is proved.

\section{REFERENCES}

1. D. Ż. Djoković, A representation theorem for $\left(X_{1}-1\right)\left(X_{2}-1\right) \ldots\left(X_{n}-1\right)$ and its applications, Ann. Polon. Math. 22 (1979), 189-198.

2. R. Ger, Mazur's criterion for continuity of convex functionals, Talk given at the 25 th ISFE in Hamburg-Rissen, 1987.

3. I. Labuda and R. D. Mauldin, Problem 24 of the "Scottish Book" concerning additive functionals, Colloq. Math. 48 (1984), 89-91.

4. Z. Lipecki, On continuity of group homomorphisms, Colloq. Math. 48 (1984), 93-94.

5. L. Székelyhidi, Regularity properties of polynomials on groups, Acta Math. Hungar. 45 (1985), 15-19.

6. _ Regularity properties of exponential polynomials on groups, Acta Math. Hungar. 45 (1985), 21-26.

7. The Scottish book, Boston, Mass., 1981.

Mathematical Institute of Lajos Kossuth University, H-4010 Debrecen, Pf. 12, HunGARY

Current address: Mathematisches Seminar der Universität Hamburg, Bundesstr. 55, D-2000 Hamburg 13, West Germany 